\title{
An Effective Data Distribution Algorithm
}

\author{
Keming Tang ${ }^{1}$, Yong $\mathrm{Xu}^{2}$, Hao Yang ${ }^{1,{ }^{*}, \mathrm{a}}$, Weipeng $L v^{1}$, Yue Ye ${ }^{1}$ \\ ${ }^{1}$ Yancheng Teachers University, Yancheng, P.R.China \\ ${ }^{2}$ Yangzhou University, Yangzhou, P.R.China \\ aEmail: classforyc@163.com
}

Keywords: Data Distribution, Algorithm, Energy-efficient.

\begin{abstract}
This paper proposes an effective data distribution mechanism based on RPL to maximize the survival times of sensors. For balancing energy consumption of sensors, an optimal data distribution algorithm is presented. The results show that the presented mechanism prolongs the survival time of network and reduces the end-to-end delay.
\end{abstract}

\section{Introduction}

Recently, Wireless Sensor Network (WSN) has displayed its effective functions in many fields, such a smart house[1], environment surveillance[2], intelligent buildings[3]. How to effectively distribute data has been paid more and more attention, especially for the low power and lossy network. IETF's ROLL group has proposed RPL[4] (Routing Protocol for Low-Power and Lossy Networks) to availably manage data traffic.

This paper proposes an algorithm of adaptive multipath traffic loading based on PRL. Its basic idea is to distribute traffic though multipath adaptively according to network's real situation. It can avoid the appearance of bottleneck node by balancing the nodes' energy, which aims at ensuring the network energy to be balanced and reducing the delay of end-to-end.

\section{Our methodology}

This section presents an effective multipath data distribution method based on RPL. In our work, we realize the entire network's energy balance by evaluating the energy consumption of bottleneck nodes in the network.

To estimate the distribution of bottleneck nodes' energy consumption precisely, this paper gives the measurement of energy distribution equilibrium degree based on Pareto's evaluation plan[5].

The definition of energy of dispersion: Assume the rational number set $A=\left\{a_{1}, a_{2}, \cdots a_{n}\right\}$ represents sensor energy, $a_{i} \geq 0$, and the ED criteria can be:

$$
E D=\sum_{i=1}^{\mathrm{n}} \varpi_{i} \cdot a_{i}^{2}
$$

Here, $\varpi_{i}=\frac{1 /\left(a_{i}-E(A)\right)^{2}}{1 / D(A)}, D(A)=\|A-E(A)\|_{2} / n$.

Futhermore, nodes will separate traffic on every path available to guarantee the lifetime of each bottleneck node is balanced. To maximize the network lifetime, we need to assign weights to each parent node to obtain the optimal solution.

This paper proposes a quick algorithm based on the metric mentioned above. Firstly, we convert metric ED to objective function $\left(E D=f\left(a_{x_{1}, c_{1}}, a_{x_{2}, c_{2}}, \cdots\right)\right)$ about $a_{x, c}$ depend on correlative math knowledge, where $c_{i}$ belong to bottleneck node set, and $x_{i}$ belong to descendants of $c_{i}$. The function is the two order continuous differentiable function, so we can solve this question by linear programming. Then, we combine Newton's as well as steepest descent method to propose one modified algorithm. This method could find one of the best group's weights to send traffic by iteration test. At the same time, it can realize convergence as soon as possible and has quite well 
stability and little calculation. Algorithm 2 gives its formal description: the nodes decide whether it gets proper answer (Line1), if not, then judge whether it is able to go specific search (Line 2). If it could then the direction would be $-\frac{\nabla f\left(x_{k}\right)}{\nabla^{2} f\left(x_{k}\right)}$ (Line 3). If couldn't then use negative gradient search direction $-\nabla f\left(x_{k}\right)$ (Line 5). Then, change the step length (Line 7), acting on iteration (Line 8), until we get the optimum solution.

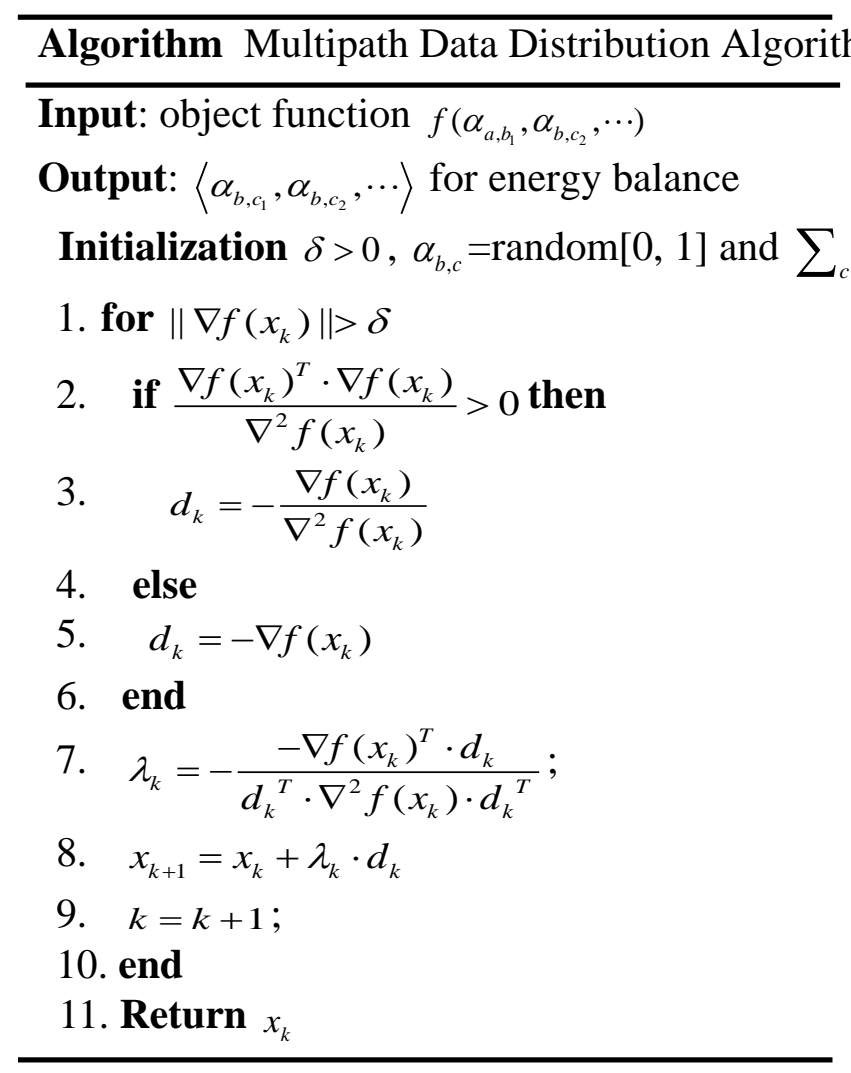

The advantage of this algorithm is the adjustment of iteration step length takes specific search on minimization of quadratic function method. The convergence rate is linear and can make sure it is every time. When the Hessian matrix's (constructed by ) maximum eigenvalue and minimum eigenvalue are close to each other, the drop speed achieve the top. Particularly, when they are equal to each other, we can obtain the optimum solution just through one iteration. The number of bottleneck nodes would change with the network. When the quantity increases, the energy consumption of $\mathrm{x}$ solution increases. At this time, we can use Quasi-Newton's method to reduce the amount of calculation about Hessian matrix, namely,

$$
\nabla^{2} f\left(x_{k+1}\right)=\nabla^{2} f\left(x_{k}\right)+\frac{s_{k}\left(s_{k}\right)^{T}}{\left(s_{k}\right)^{T} y_{k}}\left[1+\frac{\left(y_{k}\right)^{T} \cdot \nabla^{2} f\left(x_{k}\right) \cdot y_{k}}{\left(s_{k}\right)^{T} y_{k}}\right]-\frac{1}{\left(s_{k}\right)^{T} y_{k}}\left[s_{k}\left(y_{k}\right)^{T}+\left(s_{k}\right)^{T} y_{k}\right] \cdot \nabla^{2} f\left(x_{k}\right)
$$

where

$$
s_{k}=x_{k+1}-x_{k}, y_{k}=\nabla f\left(x_{k+1}\right)-\nabla f\left(x_{k}\right) .
$$

\section{Analysis of the performance}

This section evaluates the performance of our algorithm. It mainly proves the network performance (load distribution, network lifetime).

First, we compare the distributions of network load with different buffer sizes in nodes. Data package is sent every five minutes and the sink node is at the center of the network. The rest of the 
consumption is shown in the following figures. The node cache size of figure 7 is 30 bytes. The node cache size of Figure 8 is 50 bytes. From the figures we can see that sensor nodes that are closer to the data collector always have heavier workload than those edge nodes. This phenomenon can be recognized from the obvious peak of the figure. It means larger energy consumption. This is conformed to the characteristic of transmission convergence of sensor networks, because all the traffic is leading to the sink node.
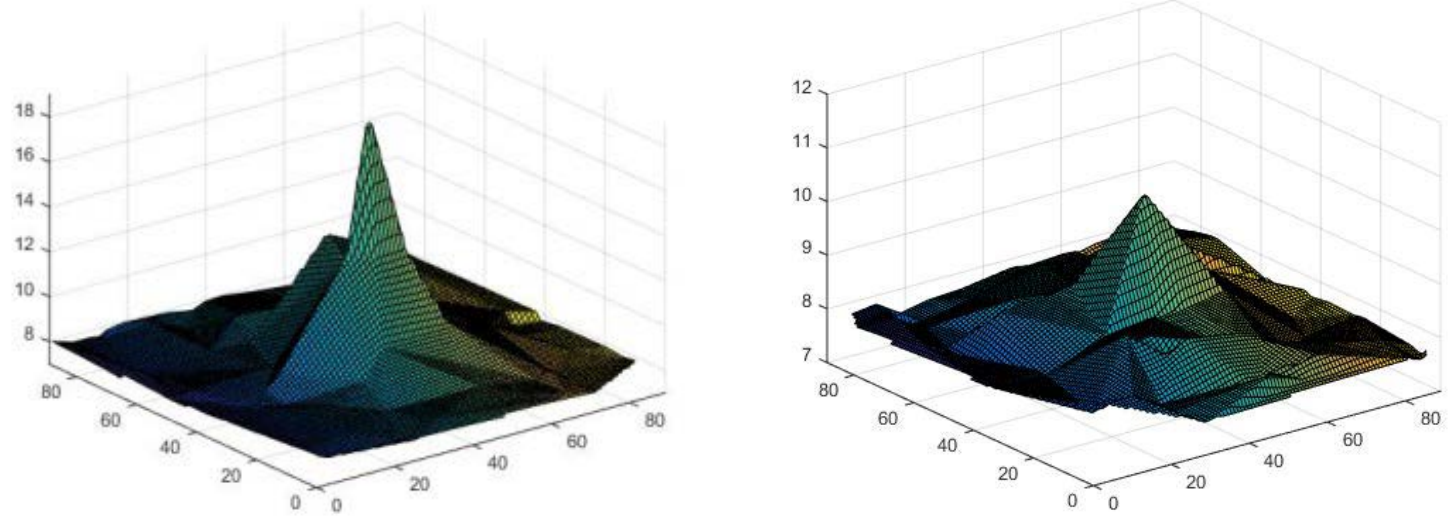

Figure 1. Residual energy distribution for RPL and our algorithm (cache size 30)
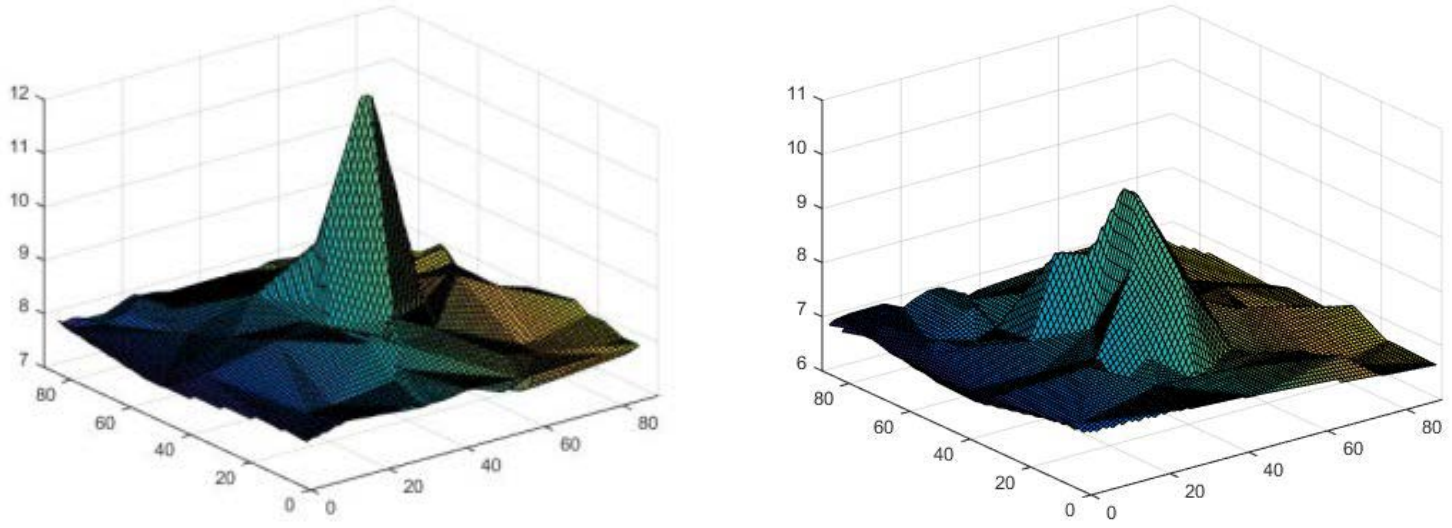

Figure 2. Residual energy distribution for RPL and our algorithm (cache size 50)

As is shown in the figures, the network lifetime becomes longer when we use energy balance to make the distribution of the energy more uniform. At this time, there's no need to consider about the energy consumption of the sink node, since the sink node usually uses the mains-powered system instead of the battery-powered system. Futhermore, the curve of result for our algorithm is smooth. It shows that the load of nodes is almost the same if they are of the same distance to the sink node. However, RPL cannot ensure this. That is, our algorithm can efficiently balance the load of the packets which are sent by the network. It can better ensure the load balance if there are less nodes. In practice, as time goes, there will be some bottleneck ports gradually arising. Their processing speed will be slowing down obviously, resulting in the gradual accumulation of the cache of surrounding nodes.

Furthermore, we validate the longest lifetime using different network sizes. Figure 2 shows that our algorithm can gain longer lifetime. This advantage becomes clearer especially when the network size is not large. Our algorithm can provide the longer lifetime. It is mainly because when the energy balance reaches a certain threshold, the node will choose the multi path forwarding according to the cache utilization ratio, and the new energy imbalance will occur. However, it will certainly do the energy balance algorithm again, which makes sure their differences will not become that large. What should be paid into attention is that the network lifetime will sharply go down by the increasing 
number of network nodes. The nodes around the sink node have to use a lot of energy to transmit the data. By this moment, none of these three algorithms can greatly reduce energy consumption.

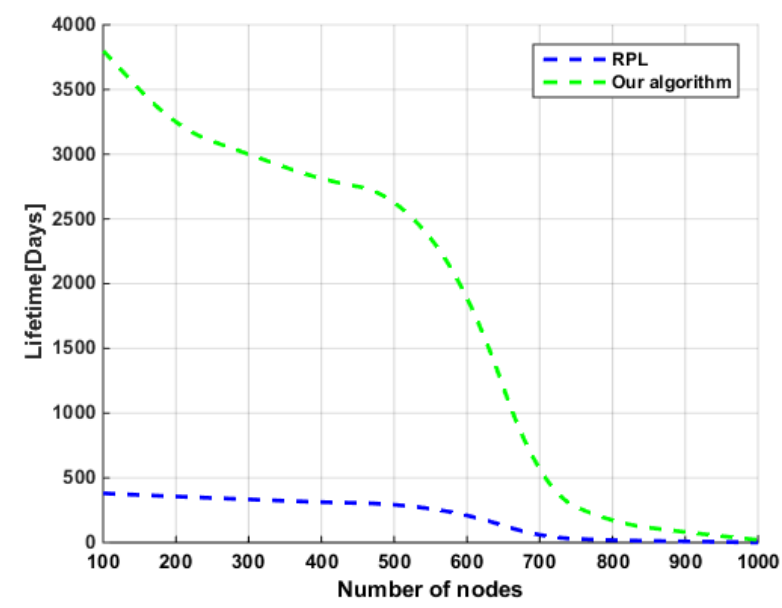

Figure 3. network lifetime in different size

\section{Summary}

In this paper, we propose an effctive multipath data distribution algorithm based on RPL to maximize meet the needs of energy consumption. Experimental results indicate that our scheme improve the network lifetime and decrease the times of DAG resetting.

\section{Acknowledgments}

The authors acknowledge the support provided by National Science Foundation of China under Grant No. 61402394, 61379064, 61273106, National Science Foundation of Jiangsu Province of China under Grant No. BK20140462, Natural Science Foundation of the Higher Education Institutions of Jiangsu Province of China under Grant No. 14KJB520040, 15KJB520035, China Postdoctoral Science Foundation funded project under Grant No. 2016M591922, Jiangsu Planned Projects for Postdoctoral Research Funds under Grant No. 1601162B, JLCBE14008,Industry university research project in Jiangsu Province under Grant No.72661632205A, Prospective joint research project of Jiangsu Province under Grant No. BY2016066-04, and sponsored by Qing Lan Project.

\section{References}

[1] K.F. Li, "Smart home technology for telemedicine and emergency management", Journal of Ambient Intelligence \& Humanized Computing, vol.4, no.5, pp.535-546, 2015.

[2] Hao Yang, Keming Tang, Jianjiang Yu, Licai Zhu, “A Practical Information Coverage Approach in Wireless Sensor Network,” Information Processing Letters, vol. 115, no. 1, pp. 6-10, 2015

[3] Hao Yang, Keming Tang, Xu Hua, Licai Zhu, Xiwei Wang, Kun Qian, “An adaptable CS-based transmission scheme validated on the real-world system,” INFOCOM 2016

[4] P. Thubert, T. Winter, A. Brandt, J. Hui, R. Kelsey, P. Levis, et al., "RPL: IPv6 Routing Protocol for Low-Power and Lossy Networks", RFC 6550, Internet Engineering Task Force, 2012,

[5] O. Gaddour, A. Koubâa, M. Abid, "Quality-of-service aware routing for static and mobile IPv6-based low-power and lossy sensor networks using RPL", Ad Hoc Networks, vol.33, pp.233-256, 2015. 